\title{
A Healthy Liver After SVR: Food for Thought
}

\author{
Brittany Rocque ${ }^{1} \cdot$ Jeffrey Kahn ${ }^{2} \cdot$ Juliet Emamaullee $^{1}$
}

Published online: 12 August 2020

(c) Springer Science+Business Media, LLC, part of Springer Nature 2020

Hepatitis $\mathrm{C}$ virus (HCV), estimated to affect 71 million people globally [1], is a leading risk factor for the development of cirrhosis and hepatocellular carcinoma (HCC) $[2$, 3]. With the introduction of direct acting antiviral (DAA) therapies over the past decade, virtually all patients can now be expected to achieve sustained virologic response (SVR), with few side effects when compared with prior interferon (IFN) and ribavirin-based regimens. These agents also reduce the risk of developing HCC [4]. Achieving SVR in patients with $\mathrm{HCV}$, regardless of the therapeutic modality, decreases the risk of liver disease-related complications including bridging fibrosis, HCC, and overall mortality [5]. Even though there are demonstrable outcome benefits associated with the eradication of detectable virus, there are still patients who demonstrate continued chronic inflammation on biopsy and patients who progress to liver disease-related complications. Questions with particular merit would be: what drives this continued disease process? and do patient habits, including substance use patterns and nutritional intake, measurably impact patient outcomes?

In this issue of Digestive Diseases and Sciences, Ohfuji and colleagues sought to identify modifiable lifestyle factors that might be protective, or alternatively, confer increased risk for the incidence of liver disease in a population of patients with HCV who achieved SVR [6]. A cohort of 180 patients with known SVR (either following a course of IFN- or DAA-based therapy) were followed prospectively after completing a detailed health questionnaire in 2005. Clinical information was collected through chart review between 2005 and 2017, with primary outcomes including

Juliet Emamaullee

Juliet.emamaullee@med.usc.edu

1 Division of Abdominal Organ Transplantation and Hepatobiliary Surgery, Department of Surgery, University of Southern California, 1510 San Pablo Street, Suite 412, Los Angeles, CA 90033, USA

2 Division of Gastrointestinal and Liver Diseases, Department of Medicine, University of Southern California, Los Angeles, CA, USA the development of cirrhosis, HCC, or liver disease-related death. Not surprisingly, the authors found that alcohol intake at the time of survey was associated with an increased risk of developing liver-related complications. Age and aspartate aminotransferase-to-platelet radio index score (APRI) $\geq 1.0$, which was used as a surrogate marker indicating the degree of HCV-related fibrosis, were also notable risk factors. These findings suggest that, even after maintaining SVR, ongoing liver injury with alcohol use can increase the likelihood of an inferior liver-related outcome. Although the actual cellular mechanism of hepatocarcinogenesis may differ between HCV-related insults of inflammation, necrosis, and regeneration cycles and alcohol- or age-related liver injury, these data suggest that these risk factors might be cumulative and that more detailed attention should be paid to the how the integration of lifestyle factors can contribute to the successes, or lack thereof, of HCV therapy.

Nutritional intake in these study subjects was also assessed. A validated questionnaire for quantifying intakes of numerous micro- and macronutrients was administered for a single time-point analysis at the time of subject enrollment in 2005 [7]. The authors found that higher intake of vitamin B12 was associated with a trend toward protection against the occurrence of cirrhosis, HCC, and liver diseaserelated mortality (HR $0.41,95 \%$ CI $0.14-1.17, p=0.09$ ). This was somewhat unexpected, particularly because the overall sample size was small $(n=180$ total subjects and only 27 subjects progressed to the complications of interest), combined with the fact that nutritional intake would be expected to vary widely across individuals. It is conceivable that an adequately powered study would ultimately yield a different result pertaining to vitamin B12 intake and reduced risk of progression to cirrhosis, HCC, and other liver-related complications. In looking back at the study design, the mere snapshot of calculated vitamin B12 intake as a result of a self-administered questionnaire does yield a trend toward reduced risk of progression of liver disease. One might suspect that over a 12-year period, the nutritional intake of an individual patient could vary over time. It would be very 
interesting to note if additional time points could corroborate the trends outlined in this study.

To add to this story of vitamin B12 supplementation and prevention of liver injury, Isoda et al. observed that vitamin B12 supplementation was hepatoprotective, specifically by reducing fibrinogenesis, in a murine model of chronically liver injury [8]. This supports the findings in this study, and while the mechanism for this observed phenomenon is unknown, it may be related, in part, to the ability of vitamin B12 to reduce oxygen free radical-mediated cellular damage [9].

There is also a growing body of literature demonstrating increased serum vitamin B12 levels in patients with chronic liver disease and HCC $[10,11]$. Some studies have even investigated serum vitamin B12 levels as a novel tumor marker for HCC and as a prognostic sign correlating to advanced disease [11, 12]. Ultimately, it is unclear how these described phenomena relate to the observation that increased nutritional intake of vitamin $\mathrm{B} 12$ is protective against liver injury. In fact, serum vitamin B12 levels do not correlate with nutritional intake in patients with HCC [12]. A potential explanation for these findings is that vitamin $\mathrm{B} 12$ is stored and metabolized in large quantities in the liver, and a passive consequence of hepatocellular injury and inflammation is increased release of cobalamin into the circulation. It remains to be seen if this is an inadvertent or controlled release of B12 by the liver during periods of inflammation and progressive injury.

The authors here used a unique approach to define lifestyle factors which may increase or reduce risk of progression of liver disease after successful treatment for HCV. Their results support future studies directed toward lifestyle modification that would aim to reduce progression of liver disease. These interventions could be as simple as providing alcohol cessation and/or nutritional counseling for patients receiving treatment for $\mathrm{HCV}$ or implementing the use of vitamin B12 supplementation as an adjunct treatment. Ongoing studies to increase the sample size are required to confirm their findings.

While this study was limited in its ability to draw certain conclusions about lifestyle factors an HCV treatment, it does provide a reminder of several important points: first, $\mathrm{HCV}$ is a large-scale public health issue; therapies for the treatment of HCV, either with interferon-based or DAA therapy, confer superior outcomes for patients. Efforts should be made to make these therapies available to patients as early as possible in their disease course in order to obtain maximal benefits. Second, the extent of liver injury again appears to be related to a cumulative effect of several insults (i.e., the damage sustained by HCV alone is less than damage sustained by HCV+ alcohol intake). Third, there should be continued study of the relative impact of nutritional supplementation on chronic liver disease, as this organ is deeply integrated with the body's metabolic functions and processing of macro- and micronutrients. Addressing these points will undoubtedly have important clinical implications, and therapies developed as a result of these investigations could potentially be low cost and high yield.

\section{References}

1. Geneva, S. W. H. O. Progress report on access to hepatitis C treatment: focus on overcoming barriers in low- and middle-income countries, March 2018. Who (2018) doi: Licence: CC BY-NC-SA 3.0 IGO.

2. Forner A, Reig M, Bruix J. Hepatocell Carcinoma. Lancet. 2018. https://doi.org/10.1016/S0140-6736(18)30010-2.

3. Ohishi W, Oka M, Mukoubayashi C, et al. Risk factors for hepatocellular carcinoma in a Japanese population: a nested casecontrol study. Cancer Epidemiol Biomark Prev. 2008. https://doi. org/10.1158/1055-9965.EPI-07-2806.

4. Asahina Y, Izumi N, Hiromitsu K, et al. JSH guidelines for the management of Hepatitis C Virus infection: a 2016 update for genotype 1 and 2. Hepatol Res. 2016. https://doi.org/10.1111/ hepr. 12645.

5. Nishiguchi S, Shiomi S, Nakatani S, et al. Prevention of hepatocellular carcinoma in patients with chronic active hepatitis C and cirrhosis. Lancet. 2001. https://doi.org/10.1016/S0140 -6736(00)03595-9.

6. Ohfuji S, Matsuura T, Tamori A, et al. Lifestyles associated with prognosis after eradication of hepatitis $\mathrm{C}$ virus: a prospective cohort study in Japan. Dig Dis Sci. (Epub ahead of print). https:// doi.org/10.1007/s10620-020-06475-0.

7. Kobayashi S, Honda S, Murakami K, et al. Both comprehensive and brief self-administered diet history questionnaires satisfactorily rank nutrient intakes in Japanese adults. J Epidemiol. 2012. https://doi.org/10.2188/jea.JE20110075.

8. Isoda K, Kagaya N, Akamatsu S, et al. Hepatoprotective effect of vitamin B12 on dimethylnitrosamine-induced liver injury. Biol Pharm Bull. 2008. https://doi.org/10.1248/bpb.31.309.

9. van de Lagemaat EE, de Groot LCPGM, van den Heuvel EGHM. Vitamin B 12 in relation to oxidative stress: a systematic review. Nutrients. 2019. https://doi.org/10.3390/nu11020482.

10. Simonsen K, Rode A, Nicoll A, et al. Vitamin B12 and its binding proteins in hepatocellular carcinoma and chronic liver diseases. Scand J Gastroenterol. 2014. https://doi.org/10.3109/00365 521.2014.921325.

11. Port GZ, Oliveira K, Soldera J, Tovo CV. Biochemical nutritional profile of liver cirrhosis patients with hepatocellular carcinoma. Arq Gastroenterol. 2014. https://doi.org/10.1590/s0004-28032 014000100003.

12. Lin CY, Kuo CS, Lu CL, Wu MY, Huang RFS. Elevated serum vitamin B 12 levels in association with tumor markers as the prognostic factors predictive for poor survival in patients with hepatocellular carcinoma. Nutr Cancer. 2010. https://doi. org/10.1080/01635580903305334.

Publisher's Note Springer Nature remains neutral with regard to jurisdictional claims in published maps and institutional affiliations. 